\title{
Formação graduada em Terapia Ocupacional na América Latina: mapeando quem somos e onde estamos*
}

\section{Undergraduation in Occupational Therapy in Latin America: mapping who we are and where we are}

\author{
Pamela Cristina Bianchi ${ }^{1}$, Ana Paula Serrata Malfitano ${ }^{2}$
}

http://dx.doi.org/10.11606/issn.2238-6149.v28i2p135-146

Bianchi PC, Malfitano APS. Formação graduada em Terapia Ocupacional na América Latina: mapeando quem somos e onde estamos.RevTerOcupUnivSãoPaulo.2017maio-ago.;28(2):135-46.

RESUMO: O presente trabalho visa refletir sobre a formação graduada em terapia ocupacional nos países latino-americanos. Partindo de um mapeamento nos websites de 114 escolas que apresentam o curso, o estudo elaborou uma análise acerca dos anos de criação dos cursos, tempo de formação, titulação dos docentes e grades curriculares. Os resultados apontaram que, embora haja um crescimento das ofertas formativas nos anos 2000, a profissão ainda é incipiente na região frente ao pequeno número de cursos de graduação, alguns de caráter técnico, e ofertado por grande número de docentes sem formação pós-graduada. Desenha-se como desafio para a terapia ocupacional latino-americana a construção de novos cursos, o aumento do número de profissionais para a conquista de campos de atuação, o desenvolvimento de pesquisas e a construção de conhecimentos próprios que contribuam com uma maior institucionalização da profissão na América Latina.

DESCRITORES: Terapia ocupacional/educação; Educação superior; América Latina/etnologia; Currículo/tendências; Instituições acadêmicas.
Bianchi PC, Malfitano APS. Undergraduation in Occupational Therapy in Latin America: mapping who we are and where we are. Rev Ter Ocup Univ São Paulo. 2017 May-Aug.;28(2):135-46.

ABSTRACT: This paper aims to reflect on undergraduate education in occupational therapy in Latin American countries. From a mapping on the websites of 114 schools that present the undergrad course, the study produced an analysis about the years of creation of courses, education time, teachers and professors degree and curricula. The results showed that although there is an increase in the number of courses in 2000 decade, the profession is still incipient in the region because the small number of undergraduate courses, some they are not higher education, and offered by a large number of teachers without $\mathrm{PhD}$. It is a deal for the Latin American Occupational Therapy the creation of new courses, increasing the number in different fields, research development and the construction of own knowledge contributing to greater institutionalization of the profession in Latin America.

KEYWORDS: Occupational therapy/education; Education, higher; Latin America/ethnology; Curriculum/trends; Schools.

\footnotetext{
*As reflexões levantadas derivam da dissertação de mestrado intitulada "Terapia ocupacional e a questão social: retratos da formação graduada a partir de um recorte latino-americano" elaborada por Pamela Cristina Bianchi sob orientação da Prof. Dra. Ana Paula Serrata Malfitano.

1. Doutoranda e Mestre em Terapia Ocupacional pelo Programa de Pós-graduação em Terapia Ocupacional, Universidade Federal de São Carlos - UFSCar, São Carlos, SP. E-mail: pamelacbianchi@gmail.com

2. Pós-doutora pela Faculdade de Terapia Ocupacional da Western University Ontario, Canadá. Professora Associada do Departamento de Terapia Ocupacional e do Programa de Pós-graduação em Terapia Ocupacional, Universidade Federal de São Carlos - UFSCar, São Carlos, SP. E-mail: anamalfitano@ufscar.br

Fonte de Financiamento: Coordenação de Aperfeiçoamento de Pessoal de Nível Superior (CAPES).

Endereço para correpondência: Laboratório METUIA, Departamento de Terapia Ocupacional, Universidade Federal de São Carlos. Rod. Washington Luís, Km 235, SP-310. CEP: 13565-905, São Carlos, SP, Brasil.
} 


\section{INTRODUÇÃO}

I ndustrialização e urbanização crescentes, implementação de políticas de seguridade social, emergência em sanar efeitos de grandes epidemias e incentivo de programas de reabilitação estrangeiros foram fatos históricos tidos como instauradores dos primeiros cursos de formação em terapia ocupacional no território latino-americano ${ }^{1,2,3}$.

Com caráter tecnicista e auxiliada por órgãos e recursos humanos estrangeiros ${ }^{1,2,3}$, os primeiros cursos de terapia ocupacional surgiram nos anos de 1950, em nível técnico na Argentina, Brasil, México e Venezuela, seguidos por Chile e Colômbia nos anos de 1960 e pelo Peru na década de $1970^{2,4}$. Os anos de 1980 foram marcantes para a profissão, visto que a maioria dos programas se reconfigurou como nível superior ao incorporar pesquisas investigativas como requisito para conclusão do curso $^{4}$. No Brasil, a instauração da formação seguiu caminhos diferentes, o qual se consolidou como nível superior no ano de $1969^{5}$.

De acordo com Testa $^{2}$, estudos sobre o desenvolvimento dos cursos de graduação em terapia ocupacional na maioria dos países da região, com exceção do Brasil, são escassamente explorados, demonstrando-se através de dados provisórios e sem aprofundamento do tema.

Em outubro de 2011, os debates realizados no Encontro Latino-Americano de Escolas e Docentes de Terapia Ocupacional tornaram evidentes que, embora o processo formativo já ocorra na região há mais de 60 anos, o cenário latino-americano ainda se encontra centrado na criação, desenvolvimento e manutenção dos cursos de graduação na área ${ }^{6}$.

Estima-se que a formação graduada em terapia ocupacional esteja presente hoje (2017) em universidades da Argentina, Bolívia, Brasil, Chile, Colômbia, Costa Rica, Equador, Guatemala, México, Panamá, Peru, Porto Rico, Uruguai e Venezuela, somando cerca de 114 cursos de graduação em instituições públicas e privadas. Além de programas de pós-graduação, especifícos da terapia ocupacional ou de áreas similares, alocados no Brasil, Chile, Colômbia e México ${ }^{7,8}$.

Passadas seis décadas, ditaduras militares, processos de democratização, implantação de políticas neoliberais, frágeis políticas sociais e expansão do ensino superior, os terapeutas ocupacionais se encontram em um período histórico de crescimento, uma época caracterizada por avanços notáveis, porém também por grandes contradições e injustiças sociais ${ }^{9}$.
Frente ao cenário apresentado, tomamos a dinâmica deste período como um convite para refletir sobre as propostas formativas assumidas pela profissão na região. Onde estão alocados os programas de formação graduada para a profissão? São cursos públicos ou privados? Houve grandes expansões? Há avanços na formação de pesquisadores e produção de conhecimentos específicos? Como são organizadas as grades curriculares dos cursos?

Partindo destes questionamentos, traçamos como objetivo do estudo mapear os cursos e conhecer as propostas de formação graduada em terapia ocupacional nos países da região latino-americana, visando ao desafio do crescimento e maior institucionalização acadêmica da profissão na região.

\section{PROCEDIMENTOS METODOLÓGICOS}

Desenvolvida entre os anos de 2015 e 2016, a pesquisa lançou mão de um estudo exploratório como estratégia de mapeamento das ofertas de formação graduada em terapia ocupacional na América Latina, bem como a apreensão de suas propostas em desenvolvimento.

Realizou-se um mapeamento dos cursos de graduação em terapia ocupacional na região, utilizandose como fontes primárias: dados fornecidos no Catálogo Latinoamericano de Asociaciones Carreras y Postgrados de Terapia Ocupacional ${ }^{7}$; informações disponibilizadas pelo Cadastro e-MEC, base de dados oficial relativa às Instituições de Ensino Superior (IES) brasileiras; e dados levantados durante a elaboração do trabalho: revisão bibliográfica referente às produções de terapeutas ocupacionais latino-americanos ${ }^{8,10}$ e pesquisa assistemática em anais de eventos científicos da área nos países latinos, como o IX Congresso Argentino de Terapia Ocupacional, realizado em Entre Ríos na província de Paraná, Argentina, em setembro de 2015.

A estratégia de coleta de dados foi a consulta aos websites de todas as universidades latinoamericanas que contém cursos de formação graduada em terapia ocupacional. O mapeamento procedeu à análise das informações organizadas em roteiro previamente elaborado pelas pesquisadoras a respeito da estrutura dos cursos, como: ano de criação, caráter de financiamento, titulação dos docentes, tempo de formação e grades curriculares.

Foram consultados os websites das 114 universidades latino-americanas, sendo elas: 13 da Argentina, 41 do Brasil, uma da Bolívia, 30 do Chile, dez da Colômbia, uma da Costa Rica, uma do Equador, uma da Guatemala, nove 
do México, uma do Panamá, uma do Peru, uma de Porto Rico, três da Venezuela e uma do Uruguai.

As informações encontradas foram tabeladas em um roteiro previamente elaborado e analisadas à luz de referenciais teóricos sobre ensino superior na América Latina e autores próprios da terapia ocupacional. Objetivouse também, no decorrer da análise, a proposição de um diálogo entre os dados referentes ao cenário internacional e à realidade brasileira, a qual estamos inseridas, visando à construção de críticas e reflexões.

\section{RESULTADOS E DISCUSSÃO}

\section{Desenvolvimento acadêmico na terapia ocupacional latino-americana}

Os anos de criação dos cursos apresentam um panorama acerca da constituição da profissão na América Latina. Tal informação foi encontrada em websites de 73 das 114 escolas investigadas, 64\% do número total. Os dados são demonstrados no Quadro 1.

Quadro 1 - Instituições de ensino superior (IES) latino-americanas que possuem o curso de graduação em terapia ocupacional

\begin{tabular}{|c|c|c|c|c|}
\hline Universidade & País & $\begin{array}{l}\text { Ano de } \\
\text { criação }\end{array}$ & $\begin{array}{c}\text { Caráter de } \\
\text { financiamento }\end{array}$ & $\begin{array}{c}\text { Tempo de formação } \\
\text { (em semestres) }\end{array}$ \\
\hline 1. Instituto Universitario del Gran Rosario & Argentina & 2008 & Privado & 8 \\
\hline 2. Universidad Abierta Interamericana - Buenos Aires & Argentina & - & Privado & 8 \\
\hline 3. Universidad Abierta Interamericana - Rosario & Argentina & 1996 & Privado & 8 \\
\hline 4. Universidad de Buenos Aires & Argentina & 1996 & Público & 8 \\
\hline 5. $\quad$ Universidad Nacional del Litoral & Argentina & - & Público & 9 \\
\hline 6. Universidad Nacional de La Rioja & Argentina & - & Público & 10 \\
\hline 7. Universidad Nacional de Mar del Plata & Argentina & - & Público & 10 \\
\hline 8. $\quad$ Universidad Nacional de Quilmes & Argentina & 1991 & Público & 8 \\
\hline 9. Universidad Nacional de San Martin & Argentina & $\begin{array}{l}1959 / \\
1999 \\
\end{array}$ & Público & - \\
\hline 10. Universidad del Norte Santo Tomás de Aquino & Argentina & 2003 & Privado & 8 \\
\hline 11. Universidad Nacional de Villa María & Argentina & 2012 & Privado & 10 \\
\hline 12. Universidad del Salvador & Argentina & - & - & 10 \\
\hline 13. Universidad Juan Agustín Maza & Argentina & 2013 & Privado & 8 \\
\hline 14. Universidade Estadual de Ciências da Saúde de Alagoas & Brasil - AL & 1996 & Público & 10 \\
\hline 15. Escola Bahiana de Medicina e Saúde Pública & Brasil - BA & 1972 & Privado & 7 \\
\hline 16. Universidade de Fortaleza & Brasil - CE & 1973 & Privado & 8 \\
\hline 17. Universidade de Brasília & Brasil - DF & 2008 & Público & 8 \\
\hline 18. Universidade Federal do Espírito Santo & Brasil - ES & 2009 & Público & 10 \\
\hline 19. Faculdade União de Goyazes & Brasil - GO & - & Privado & 8 \\
\hline 20. Faculdade Santa Teresinha & Brasil - MA & - & Privado & 8 \\
\hline 21. Faculdade de Minas Gerais & Brasil - MG & - & Privado & 8 \\
\hline 22. Universidade Federal do Triângulo Mineiro & Brasil - MG & 2007 & Público & 10 \\
\hline 23. Universidade Federal de Minas Gerais & Brasil - MG & 1979 & Público & 8 \\
\hline 24. Escola Superior da Amazônia & Brasil - PA & - & Privado & 8 \\
\hline 25. Universidade da Amazônia & Brasil - PA & - & Privado & 4 \\
\hline 26. Universidade do Estado do Pará & Brasil - PA & - & Público & 10 \\
\hline 27. Universidade Federal do Pará & Brasil - PA & 2009 & Público & 10 \\
\hline 28. Universidade Federal da Paraíba & Brasil - PB & 2010 & Público & 8 \\
\hline
\end{tabular}


Bianchi PC, Malfitano APS. Formação graduada em Terapia Ocupacional. Rev Ter Ocup Univ São Paulo. 2017 maio/ago.;28(2):135-46.

Quadro 1 - Instituições de ensino superior (IES) latino-americanas que possuem o curso de graduação em terapia ocupacional

\begin{tabular}{|c|c|c|c|c|}
\hline Universidade & País & $\begin{array}{l}\text { Ano de } \\
\text { criação }\end{array}$ & $\begin{array}{c}\text { Caráter de } \\
\text { financiamento }\end{array}$ & $\begin{array}{c}\text { Tempo de formação } \\
\text { (em semestres) }\end{array}$ \\
\hline 29. Universidade Federal do Paraná & Brasil - PR & 2000 & Público & 9 \\
\hline 30. União do Ensino Superior do Iguaçu & Brasil - PR & 2013 & Privado & 8 \\
\hline 31. Universidade Federal do Pernambuco & Brasil - PE & 1962 & Público & 8 \\
\hline 32. Faculdade Integral Diferencial & Brasil - PI & - & Privado & 8 \\
\hline 33. Instituto Federal de Educação Ciência e Tecnologia do Rio de Janeiro & Brasil - RJ & 2009 & Público & 8 \\
\hline 34. Universidade Federal do Rio de Janeiro & Brasil - RJ & 2009 & Público & 8 \\
\hline 35. Faculdade Serra Gaúcha & Brasil - RS & - & Privado & 8 \\
\hline 36. Centro Universitário Franciscano & Brasil - RS & 2004 & Privado & 9 \\
\hline 37. Universidade Federal de Santa Maria & Brasil - RS & 2009 & Público & 8 \\
\hline 38. Universidade Federal de Pelotas & Brasil - RS & 2010 & Público & 8 \\
\hline 39. Faculdades Integradas Aparício Carvalho & Brasil - RO & 2009 & Privado & 8 \\
\hline 40. Associação Catarinense de Ensino - Faculdade Guilherme Guimbala & Brasil - SC & 2010 & Privado & 8 \\
\hline 41. Centro Universitário de Araraquara & Brasil - SP & - & Privado & 8 \\
\hline 42. Centro Universitário São Camilo & Brasil - SP & - & Privado & 8 \\
\hline 43. Rede Claretiano & Brasil - SP & - & Privado & 8 \\
\hline 44. Faculdade de Medicina do ABC & Brasil - SP & 2008 & Privado & 8 \\
\hline 45. Faculdades Integradas de Fernandópolis & Brasil - SP & - & Privado & 8 \\
\hline 46. Pontifícia Universidade Católica de Campinas & Brasil - SP & 1977 & Privado & 8 \\
\hline 47. Universidade Sagrado Coração & Brasil - SP & 1999 & Privado & 8 \\
\hline 48. Universidade de São Paulo - São Paulo & Brasil - SP & 1956 & Público & 8 \\
\hline 49. Universidade de São Paulo - Ribeirão Preto & Brasil - SP & 2002 & Público & 10 \\
\hline 50. Universidade de Sorocaba & Brasil - SP & 1998 & Privado & 8 \\
\hline 51. Universidade Estadual Paulista "Júlio de Mesquita Filho" & Brasil-SP & 2003 & Público & 8 \\
\hline 52. Universidade Federal de São Carlos & Brasil - SP & 1978 & Público & 10 \\
\hline 53. Universidade Federal de São Paulo & Brasil - SP & 2006 & Público & 8 \\
\hline 54. Universidade Federal de Sergipe & Brasil - SE & 2011 & Público & - \\
\hline 55. Universidad Mayor de San Andrés & Bolívia & 2010 & Público & - \\
\hline 56. Universidad Santo Tomás - Arica & Chile & - & Privado & 10 \\
\hline 57. Universidad Santo Tomás - Concepción & Chile & 2010 & Privado & 10 \\
\hline 58. Universidad Santo Tomás - Osorno & Chile & - & Privado & 10 \\
\hline 59. Universidad Santo Tomás - Puerto Montt & Chile & 2011 & Privado & 10 \\
\hline 60. Universidad Santo Tomás - Santiago & Chile & - & Privado & 10 \\
\hline 61. Universidad Santo Tomás -Valdívia & Chile & 2011 & Privado & 10 \\
\hline 62. Universidad Santo Tomás - Viña del Mar & Chile & 2009 & Privado & 10 \\
\hline 63. Universidad Viña del Mar & Chile & 2011 & Privado & 10 \\
\hline 64. Universidad Andrés Bello - Concepción & Chile & - & Privado & 10 \\
\hline 65. Universidad Andrés Bello - Santiago & Chile & 2002 & Privado & 10 \\
\hline 66. Universidad Andrés Bello - Viña del Mar & Chile & - & Privado & 10 \\
\hline 67. Universidad Austral de Chile & Chile & 2004 & Privado & 10 \\
\hline
\end{tabular}


Quadro 1 - Instituições de ensino superior (IES) latino-americanas que possuem o curso de graduação em terapia ocupacional

\begin{tabular}{|c|c|c|c|c|}
\hline Universidade & País & $\begin{array}{l}\text { Ano de } \\
\text { criação }\end{array}$ & $\begin{array}{c}\text { Caráter de } \\
\text { financiamento }\end{array}$ & $\begin{array}{c}\text { Tempo de formação } \\
\text { (em semestres) }\end{array}$ \\
\hline 68. Universidad Autonoma de Chile - Santiago & Chile & - & Privado & 9 \\
\hline 69. Universidad Autonoma de Chile - Temuco & Chile & - & Privado & 9 \\
\hline 70. Universidad Central de Chile & Chile & 2010 & Privado & 10 \\
\hline 71. Universidad de Chile & Chile & 1963 & Público & 10 \\
\hline 72. Universidad de la Frontera & Chile & 2010 & Privado & 10 \\
\hline 73. Universidad de las Américas -Concepción & Chile & - & Privado & 10 \\
\hline 74. Universidad de las Américas -Providencia & Chile & - & Privado & 10 \\
\hline 75. Universidad de las Américas -Santiago & Chile & - & Privado & 10 \\
\hline 76. Universidad de las Américas - Viña del Mar & Chile & - & Privado & 10 \\
\hline 77. Universidad de los Andes & Chile & 2010 & Privado & 10 \\
\hline 78. Universidad Internacional SEK & Chile & - & Privado & 10 \\
\hline 79. Universidad de Magallanes & Chile & 2003 & Público & 10 \\
\hline 80. Universidad Mayor & Chile & - & - & 10 \\
\hline 81. Universidad de Playa Ancha & Chile & 1996 & Público & 10 \\
\hline 82. Universidad San Sebastian-Concepción & Chile & - & Privado & 10 \\
\hline 83. Universidad San Sebastian -Santiago & Chile & 2010 & Privado & 10 \\
\hline 84. Universidad Bolivariana & Chile & - & Privado & 10 \\
\hline 85. Universidad Bernardo O’Higgins & Chile & 2012 & Privado & 10 \\
\hline 86. Escuela Colombiana de Rehabilitación & Colômbia & 1969 & Privado & 8 \\
\hline 87. Universidad de Santander & Colômbia & 1995 & Privado & 10 \\
\hline 88. Universidad Manuela Beltrán - Bucaramanga & Colômbia & 1994 & Privado & 9 \\
\hline 89. Universidad Manuela Beltrán - Bogotá & Colômbia & - & Privado & 9 \\
\hline 90. Universidad Nacional de Colombia & Colômbia & 1966 & Público & 10 \\
\hline 91. Universidad de Pamplona & Colômbia & - & - & 10 \\
\hline 92. Universidad Colegio Mayor de Nuestra Señora del Rosário & Colômbia & 1969 & Privado & 10 \\
\hline 93. Universidad del Valle & Colômbia & 1986 & - & 10 \\
\hline 94. Universidad Mariana de Pasto & Colômbia & 1999 & Privado & 9 \\
\hline 95. Universidad Metropolitana & Colômbia & 1995 & Privado & 8 \\
\hline 96. Universidad Santa Paula & Costa Rica & 1994 & Privado & 8 \\
\hline 97. Universidad Central del Ecuador & Equador & - & Público & 8 \\
\hline 98. Universidad de San Carlos de Guatemala & Guatemala & - & - & - \\
\hline 99. Centro de Rehabilitacion "Gaby Brimer" & México & - & - & - \\
\hline 100. Centro Mexicano Universitario de Ciencias y Humanidades & México & 2000 & Privado & 9 \\
\hline 101. Centro de Rehabilitación y Educación Especial Puebla & México & 1989 & Privado & - \\
\hline 102. Instituto Científico de Educación Superior & México & 2006 & Privado & - \\
\hline 103. Instituto Mexicano del Seguro Social & México & - & - & - \\
\hline 104. Instituto Nacional de Rehabilitación & México & - & - & \\
\hline 105. Instituto Teleton de Estudios Superiores en Rehabilitacion & México & 2012 & Privado & 8 \\
\hline 106. Instituto de Terapia Ocupacional & México & 2009 & Privado & 8 \\
\hline
\end{tabular}


Bianchi PC, Malfitano APS. Formação graduada em Terapia Ocupacional. Rev Ter Ocup Univ São Paulo. 2017 maio/ago.;28(2):135-46.

Quadro 1 - Instituições de ensino superior (IES) latino-americanas que possuem o curso de graduação em terapia ocupacional

\begin{tabular}{|l|l|c|c|c|}
\hline Universidade & País & $\begin{array}{c}\text { Ano de } \\
\text { criação }\end{array}$ & $\begin{array}{c}\text { Caráter de } \\
\text { financiamento }\end{array}$ & $\begin{array}{c}\text { Tempo de formação } \\
\text { (em semestres) }\end{array}$ \\
\hline 107. Universidad Autónoma del Estado de México & México & 2001 & Público & - \\
\hline 108. Universidad Especializada de las Américas & Panamá & 2000 & Público & 8 \\
\hline 109. Universidad Nacional Mayor de San Marcos & Peru & - & Público & 10 \\
\hline 110. Universidad de Porto Rico & Porto Rico & 2003 & - & 4 \\
\hline 111. Universidad de la Republica Uruguay & Uruguai & 2002 & Público & 9 \\
\hline 112. Colegio Universitário de los Teques Cecílio Acosta & Venezuela & 1986 & Público & - \\
\hline 113. Colegio Universitário de Rehabilitación May Hamilton & Venezuela & - & - & 6 \\
\hline 114. Universidad Central de Venezuela & Venezuela & 1967 & Público & 8 \\
\hline
\end{tabular}

Os primeiros cursos datam das décadas de 1950, no Brasil e na Argentina, e de 1960, no Chile, Colômbia e Venezuela, sendo, portanto, estes os pioneiros na formação e institucionalização acadêmica da formação graduada em terapia ocupacional na região.

Com exceção da Colômbia, Costa Rica e Venezuela, que não apresentaram novos cursos após os anos 2000, os demais países assinalaram um aumento importante com 45 novas escolas com formação em terapia ocupacional no período entre os anos 2000 e 2013 , mais de $63 \%$, evidenciando grande expansão do ensino e do número de profissionais na região no século XXI. O Gráfico 1 ilustra os dados acima.

Gráfico 1 - Ano de criação dos cursos de terapia ocupacional nos países latino-americanos

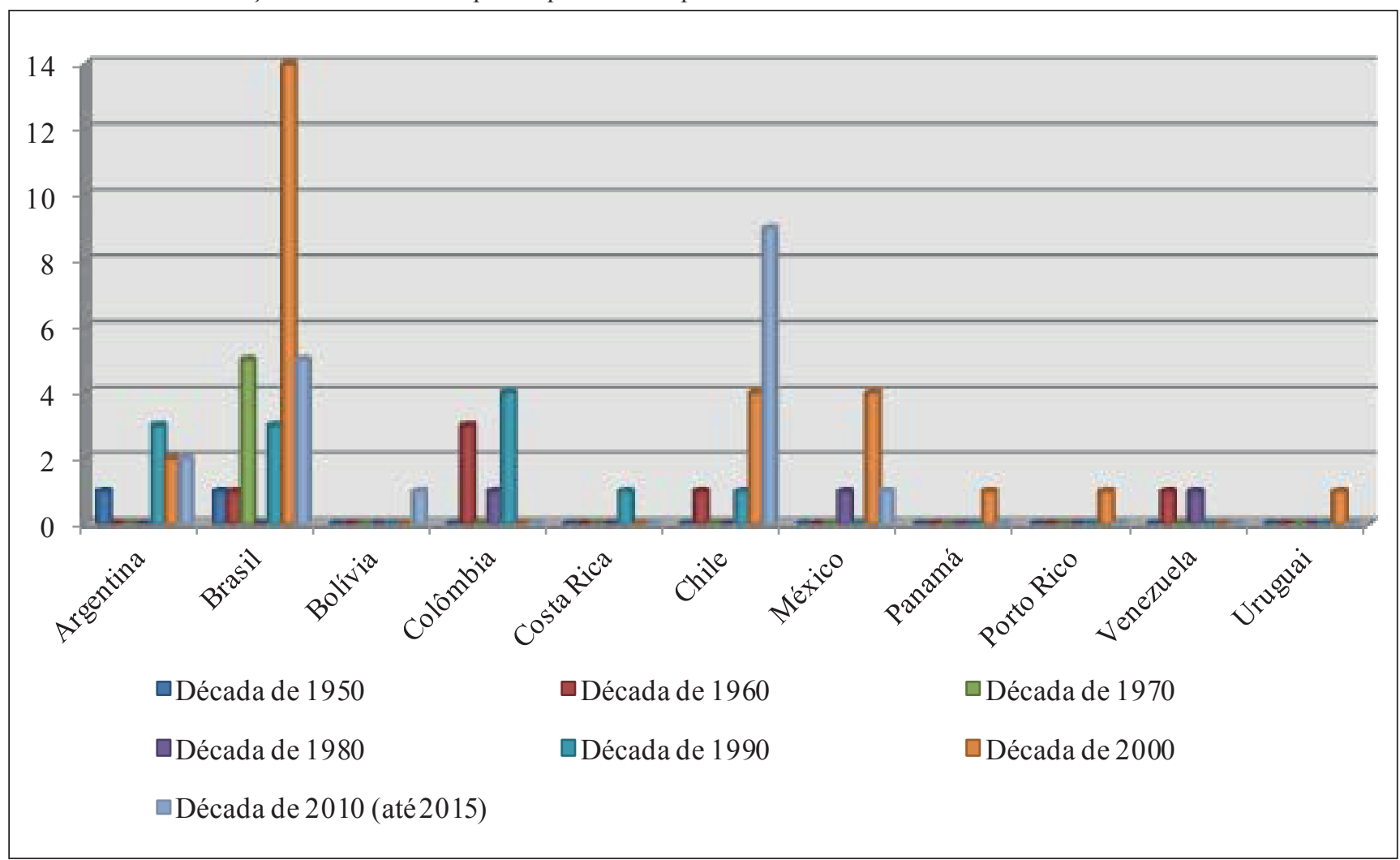


O aumento do número de cursos de terapia ocupacional acompanhou o movimento de expansão do ensino superior latino-americano. De acordo com Chiroleu $^{11}$, a tradicionalmente escassa cobertura no ensino superior da região tornou-se foco de atenção das políticas nacionais de vários países, em especial, quanto à expansão da oferta pública no início do século XXI. Projetos nacionais de diferentes formatos passaram a ser desenhados como as universidades interculturais na região Andina e no México, a expansão das universidades federais no Brasil, a municipalização da educação superior na Venezuela e a criação de universidades nacionais e centros universitários na Argentina. Em todos os casos, a expansão da oferta universitária compartilhou a geração de mais oportunidades e reconhecimento neste nível de ensino, implicando diretamente na inclusão de outros grupos sociais ao ensino superior.

No caso específico do Brasil, a grande expansão dos cursos de graduação em terapia ocupacional nas décadas de 2000 e 2010 foi fruto das políticas públicas de fomento ao ingresso no ensino superior, tanto na esfera privada quanto, e principalmente, no âmbito público.

Na esfera privada, o cenário brasileiro é complexo. As políticas de acesso ao ensino superior favoreceram à abertura de novos cursos e ao aumento de vagas em programas já existentes. Contudo, o crescimento do setor privado no Brasil é reflexo do incentivo proveniente de recursos públicos. Figueiredo ${ }^{12}$ aponta que os incentivos financeiros do governo federal destinados às universidades públicas sofreram drástica diminuição entre 2011 e 2016, em contraponto, neste mesmo período é visualizado um grande aumento do recurso público às iniciativas privadas. A crítica que circunda este movimento do governo é ilustrada com base no questionamento sobre o desinvestimento no âmbito público, prioridade estatal, e atuação para contribuição no mercado, âmbito privado. Além disso, o que se visualiza também é a ausência de mecanismos de controle da qualidade do ensino privado e de exigência de melhor qualificação de docentes, implicando na precarização do ensino, declínios de salários e rotatividade de professores ${ }^{12}$.

No âmbito público, especificamente quanto às universidades federais, lançou-se em 2007 o Programa de Apoio a Planos de Reestruturação e Expansão das Universidades Federais (REUNI), com o objetivo de criar condições para a ampliação de vagas e acesso e estimular a permanência dos alunos no ensino superior, visando ao aumento da qualidade dos cursos e melhor aproveitamento da estrutura física e recursos humanos ofertados nas instituições federais ${ }^{13}$.
Para a área de terapia ocupacional, de acordo com os dados discutidos por $\mathrm{Pan}^{14}$, com a implantação do REUNI, oito novos cursos foram criados (UnB, UFPA, UFES, UFRJ, UFSM, UFPB, UFPel e UFS), constituindose como um programa determinante para a atual expansão da profissão no país, tanto na perspectiva de aumento de número de profissionais quanto na abrangência de regiões que não possuíam cursos na área em instituições públicas. Apesar deste significativo crescimento, é preciso destacar que a demanda de formação no país não foi ainda suprida, na medida em que há estados brasileiros que não possuem esta oferta, nem no âmbito privado tampouco no âmbito público, requerendo ações da categoria para o desenvolvimento da profissão no país ${ }^{15}$. $\mathrm{O}$ crescimento nas universidades públicas, contudo, é acompanhado da ressalva que a implementação dos programas não se deu de maneira uniforme, principalmente em relação ao financiamento e à contratação de docentes, instituindo-se de forma precária e impossibilitando o pleno desenvolvimento de alguns projetos ${ }^{14}$.

\section{Caráter de financiamento e seus reflexos para a terapia ocupacional}

O caráter público e privado é um fator importante na compreensão e avaliação da qualidade de ensino, incentivo do Estado no processo formativo, fomento às pesquisas e rebatimento na prática profissional. Quando se trata de profissionais ligados diretamente à efetivação de políticas públicas, como o terapeuta ocupacional, e, portanto, de pequeno interesse lucrativo para o mercado liberal, tal característica tem impactos na oferta de cursos.

Neste concerne, sobre a relevância e diferenças de possibilidade de desenvolvimento e atenção à demanda entre o caráter público ou privado das universidades, investigou-se este item, sendo que obtivemos dados de 104 escolas, $91 \%$ do total.

Das universidades alocadas na Argentina, seis possuem natureza pública e seis privada, apenas uma escola não forneceu esta informação. No Brasil, logrou-se o número total de 20 escolas públicas e 21 privadas, com uma pequena margem percentual de prevalência do ensino privado. A única escola boliviana é pública. No Chile, as distinções aparecem em maior escala, das 29 instituições de ensino que demonstraram a informação, apenas três são de caráter público, as demais, somando 26, provém de financiamento privado. Similar à realidade chilena, a Colômbia também possui um número pequeno de universidades públicas com curso de graduação em terapia ocupacional, das oito informadas apenas uma é pública, 
sete são privadas. A Costa Rica possui uma universidade com o curso, também de caráter privado. No Equador, a única universidade com curso de terapia ocupacional no país é de nível público. Das seis universidades mexicanas que apresentaram este dado, cinco escolas são privadas e uma é pública. A única universidade do Panamá com o curso de graduação em terapia ocupacional é pública. Assim como no Uruguai, com apenas uma escola e de caráter público. $\mathrm{Na}$ Venezuela, das três escolas, duas forneceram este dado, no qual, ambas são públicas. As informações estão ilustradas no Quadro 1.

Destarte das diferenças políticas de configuração do ensino superior nos países, observa-se a predominância do ensino de âmbito privado nas escolas latinoamericanas de terapia ocupacional, visto que a maioria, 66 instituições de ensino, $63 \%$, são privadas, enquanto apenas 38 são públicas, $36 \%$.

Segundo Durham e Sampaio ${ }^{16}$, nas últimas décadas do século XX, os países da América Latina passaram pelo intenso fenômeno das privatizações, influenciando os processos de financiamento do ensino superior na região. As autoras retratam o fenômeno como resultado da influência neoliberal nas políticas e da adoção de uma lógica mercantil deletéria aos interesses do ensino e da subordinação às exigências do capitalismo e globalização do mercado.

No debate, o desígnio de privatização tem sido utilizado para ilustrar experiências distintas: a expansão das matrículas no setor privado (Brasil), sobretudo em países com uma larga tradição no ensino superior público (Argentina e México); a introdução da cobrança de taxas de matrículas e anuidade em universidades públicas (Chile no início dos anos 1980 e Colômbia nos anos 1990), a apresentação de propostas de controle no setor público de ensino superior e diferentes iniciativas de avaliação institucional ${ }^{16}$.

Outro rebatimento importante levantado pelas autoras refere-se ao fomento às pesquisas, fundamentais para a promoção da qualidade do ensino superior e construção de conhecimento. Apesar da procura e organização de algumas universidades privadas na aquisição de financiamento às pesquisas e pós-graduações, os recursos públicos voltam-se predominantemente ao incentivo e fomento de investigações do setor público, gerando maior número de pesquisadores e docentes qualificados nesta esfera de ensino ${ }^{16}$.

\section{Tempo de formação e grades curriculares: indicativos do processo formativo}

Outro dado relevante investigado no mapeamento dos cursos de terapia ocupacional foi o tempo de formação.
Cento e cinco universidades, 92\%, apresentaram informações quanto à duração dos cursos, os quais variam entre quatro e dez semestres. Pontua-se que os cursos de quatro e seis semestres possuem caráter técnico e estão presentes em Porto Rico e na Venezuela. A maioria dos cursos apresenta um tempo de duração de oito a dez semestres, configurando-se como carreiras de nível superior. O Quadro 1 ilustra os dados.

As informações referentes às grades curriculares dos cursos foram encontradas em 90 universidades, $79 \%$ do total, destas 36 escolas estão alocadas no Brasil. A maioria dos cursos, 87 escolas, apresenta-se com grades curriculares próximas quanto à estrutura, carga horária e apresentação das disciplinas, organizadas em ciclo básico e ciclo final.

O ciclo básico contempla o ensino das áreas biológicas e da saúde, sociais e humanas, psicológicas, exatas e àquelas relativas à pesquisa, salvo até três disciplinas teóricas específicas sobre terapia ocupacional, nos primeiros anos. As disciplinas relacionadas ao ensino de áreas específicas e práticas profissionais da terapia ocupacional são, na maioria, organizadas a partir do terceiro ano de graduação, compondo o ciclo final da formação.

As disciplinas voltadas à área biológica e da saúde compreendem, de uma maneira geral: biologia, anatomia, fisiologia, neuroanatomia, neurologia, patologia, imunologia, psiquiatria e farmacologia. Neste perfil curricular, estas disciplinas ocupam a maior parte da carga horária de formação quando comparadas às demais áreas do ciclo básico, com uma média de oito a dez por curso. Destacam-se os cursos chilenos que possuem, em média, 11 disciplinas de temática biológica em sua grade curricular.

As ciências humanas e sociais aparecem em pequena quantidade, cerca de duas disciplinas por curso, como: antropologia e sociologia. No Brasil, 18 escolas apresentam disciplinas voltadas à área das humanidades em interface ao campo da saúde, exemplos são "Saúde e Sociedade" e "Antropologia da Saúde".

A área de exatas também surge em pequena quantidade e se expressa através de uma ou duas disciplinas, como: matemática aplicada à saúde e biofísica. Aponta-se que um grande número de escolas brasileiras não apresenta disciplinas na área de exatas, salvo àquelas que propõem interface com a saúde, como bioestatística e epidemiologia. A área de psicologia é retratada entre duas e quatro disciplinas por curso, como: psicologia geral e psicologia do desenvolvimento.

As disciplinas relacionadas à pesquisa apresentamse em pequeno número, duas por curso em média, e algumas de ordem optativa, exemplos são: métodos de pesquisa e metodologia do trabalho científico. Já as 
específicas da terapia ocupacional, que compõe o ciclo básico com mais incidência, são: fundamentos e/ou história da terapia ocupacional e atividades e recursos terapêuticos (ART).

As disciplinas do ciclo final específicas de terapia ocupacional apresentam-se em maior número, aproximadamente 16 por curso, e se dividem entre conteúdos teóricos e práticos. Os campos abordados em tais disciplinas são: terapia ocupacional comunitária, terapia ocupacional e atenção básica em saúde, terapia ocupacional em saúde mental, terapia ocupacional em reabilitação física, terapia ocupacional em saúde do trabalhador, terapia ocupacional social e terapia ocupacional em gerontologia.

Dentre os 90 cursos que ofertaram informações a respeito de suas grades curriculares, apenas três escolas, todas brasileiras, lançam mão de outras estruturas de formação. A Universidade Federal de São Carlos utiliza-se de uma mescla de disciplinas tradicionais e metodologias ativas como estratégia formativa, visando ao desenvolvimento de competências ${ }^{17}$. O curso da Universidade Federal de Sergipe orienta-se através de ciclos anuais por competências, nos quais se visa à formação generalista do terapeuta ocupacional ${ }^{18}$. A Universidade Federal de São Paulo, por sua vez, tem uma estrutura curricular organizada em quatro eixos de formação e é pautada no ensino interdisciplinar e integrado entre cinco cursos da área da saúde ${ }^{14}$.

Nota-se que os cursos de terapia ocupacional das universidades latino-americanas analisadas possuem estrutura e áreas de formação bastante similares e com forte viés no campo biológico e da saúde, apontando grande influência biomédica na formação e lacunas no ensino das ciências sociais e humanas.

Os dados são similares aos resultados da pesquisa elaborada por $\mathrm{Pan}^{14}$, em investigação sobre as universidades federais brasileiras. A pesquisa evidenciou uma elevada porcentagem de conteúdos orientados às ciências biológicas e da saúde e uma desproporcionalidade quando comparados aos conteúdos sobre as ciências sociais e humanas. Pan $^{14}$ afirma que isto se deve às raízes históricas da profissão que, nos anos de 1960, vivenciou um movimento de alinhamento aos pressupostos do modelo biomédico com vistas a alcançar validação técnica e científica. As implicações deste processo são visualizadas até hoje na busca da legitimação e construção de uma identidade profissional.

Evidentemente, a discussão sobre a adoção do modelo biomédico na formação e, futuramente, na prática profissional não se restringe à oferta de disciplinas da área biológica, mas sim em seu rebatimento na conduta profissional adotada. Contudo, considera-se um dado objetivo para apoiar a discussão acerca da formação e vertentes predominantes.

Em suma, o campo da saúde, em um viés biomédico, ainda constitui-se como temática prioritária na formação graduada em terapia ocupacional na América Latina. Faz-se necessário, contudo, questionar a característica histórica e a emergência de novas problemáticas sociais na região e o real preparo dos profissionais para lidarem com intervenções direcionadas ao contexto latinoamericano, que demanda profissionais envolvidos com a sua realidade social.

\section{Titulação dos docentes dedicados à formação de terapeutas ocupacionais latino-americanos}

Compreendendo de antemão que a inserção majoritária dos cursos no âmbito privado limitam o desenvolvimento da pesquisa e consequente produção de conhecimento na área, buscamos dados sobre a titulação dos docentes responsáveis pelo ensino da profissão. Contudo, nomes e títulos de professores são dados com pouca especificação nos sítios eletrônicos da maioria das escolas latino-americanas, com exceção das instituições brasileiras, que disponibilizam o currículo acadêmico de seus pesquisadores em uma plataforma específica: a Plataforma Lattes, através do Currículo Lattes ${ }^{19}$. Das 114 universidades consultadas, apenas 40 escolas fornecem estas informações, aproximadamente 35\%, dentre elas, 28 estão alocadas no Brasil.

Encontrou-se um total de 456 docentes graduados em terapia ocupacional, destes: 112 em nível de doutorado; $63 \mathrm{em}$ processo de doutoramento; 160 mestres; três mestrandos; 77 em nível de especialização; e 41 profissionais não possuem pós-graduação. As informações seguem ilustradas no Gráfico 2.

Lançamos luz às universidades brasileiras em virtude de serem aquelas que maior ofertaram informações referentes à titulação de seus docentes, consequência da política nacional do ensino superior, que exige a publicização de currículos desta categoria ${ }^{19}$. Foram encontrados 337 professores terapeutas ocupacionais em aproximadamente $70 \%$ do total de cursos (28), dentre estes: 107 professores são doutores, 63 estão em processo de doutoramento, 119 são mestres, três são mestrandos, 44 especialistas, e apenas um docente não possui formação pós-graduada. Vale ressaltar que cerca de $90 \%$ do total de professores doutores estão alocados em universidades públicas. Informações apresentadas no Gráfico 3. 
Gráfico 2 - Titulação de docentes dos cursos de terapia ocupacional das universidades latino-americanas

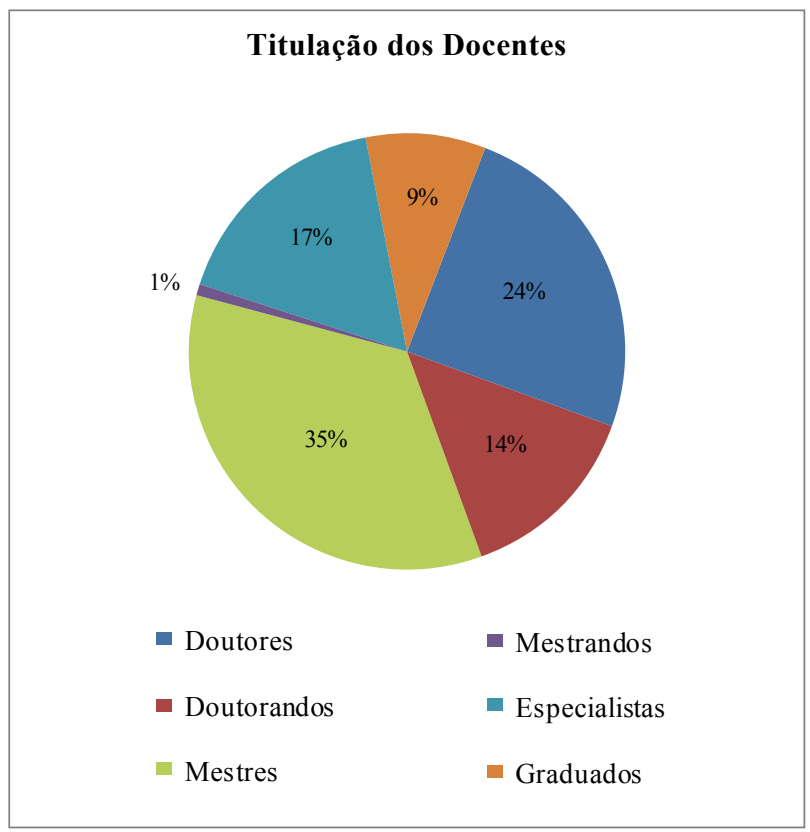

Gráfico 3 - Titulação de docentes brasileiros dos cursos de terapia ocupacional de universidades brasileiras

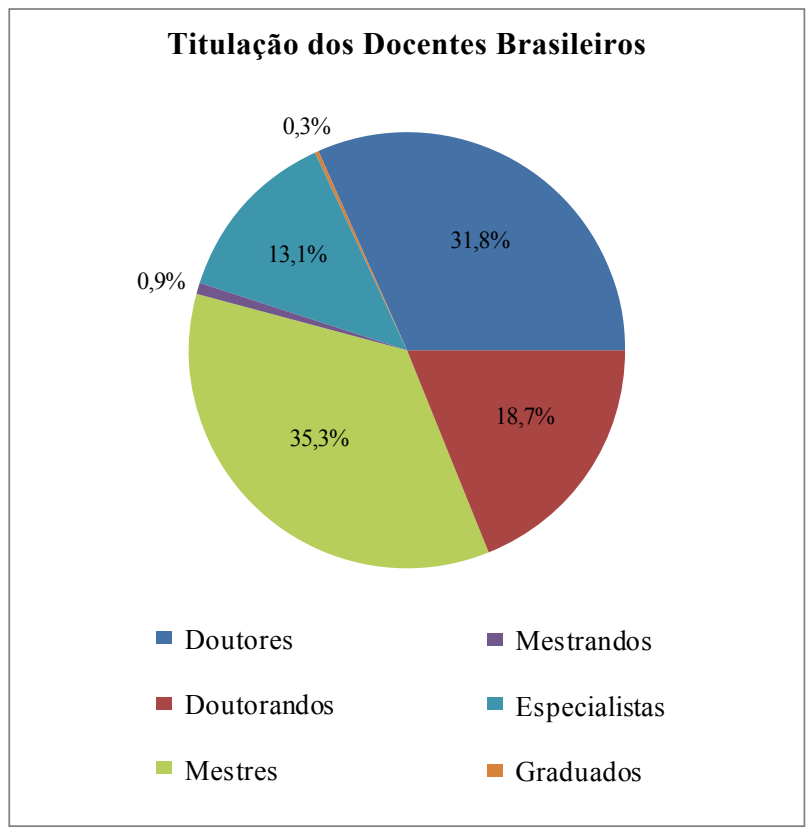

No Brasil, o índice relativamente alto de professores doutores ocorre pela exigência de maior qualificação e oferecimento de melhores condições para a carreira em universidades públicas. Neste cenário, é função do docente realizar atividades de ensino, extensão e pesquisa, assim, tais funções refletiram em um aumento significativo da titulação de doutores e na produção de conhecimento no país ${ }^{20,21}$.

A capacitação docente por meio de cursos de mestrado e doutorado ocorreu, entre docentes da área de terapia ocupacional no Brasil, no início dos anos 1990. Embora os estudos fossem realizados em programas alheios à terapia ocupacional, uma vez que não existiam programas de pós-graduação stricto sensu na área, estes possibilitaram ampliar a produção de conhecimento em interface com a profissão ${ }^{21}$.

Uma das consequências da pouca titulação de doutores na docência em terapia ocupacional na América Latina reflete no pequeno quadro de oferta de formação pós-graduada.

Nos anos 2000, pesquisadores terapeutas ocupacionais brasileiros participaram da abertura de dois cursos de pós-graduação stricto sensu na área de Ciências da Reabilitação, um na Universidade Federal de Minas Gerais $^{22}$, aprovado em 2002, e outro na Universidade de São Paulo ${ }^{23}$, aprovado em 2004. Os Programas possuem linhas de pesquisa com oferta de estudos na área ou em interface e contribuições de professores orientadores da terapia ocupacional, possibilitando a discussão e produção de conhecimentos em interface às outras áreas da reabilitação, como fisioterapia e fonoaudiologia. Contudo, a formação de pesquisadores terapeutas ocupacionais é proprorcionalmente pequena, tendo em vista o número de formados em cada programa e a demanda dos terapeutas ocupacionais.

No ano de 2010 teve início o Programa de Pósgraduação em Terapia Ocupacional na Universidade Federal de São Carlos - PPGTO/UFSCar. O programa nasceu com o objetivo de consolidar e ampliar os conhecimentos já adquiridos e formar mestres e doutores capacitados em pesquisas que retratem as bases da profissão, pautadas em temáticas como desenvolvimento humano e inserção social ${ }^{24}$. O Programa tem contribuído com a ampliação da formação stricto sensu de terapeutas ocupacionais no país.

Segundo o Catálogo Latinoamericano de Asociaciones Carreras y Postgrados de Terapia Ocupacional, além do Brasil, o Chile, na Universidad Andrés Bello, dispõe de um mestrado em Terapia Ocupacional Con Menciones, contudo não há informações atuais sobre a oferta do curso. Na Colômbia, na Universidad Nacional de Colombia, o Mestrado em Discapacidad e Inclusión Social conta com a participação de terapeutas 
ocupacionais pesquisadores. E no México há o Mestrado em Terapia Ocupacional, em funcionamento desde 2004.

Espera-se que, com o tempo, haja o crescimento de programas específicos em terapia ocupacional na região, possibilitando o fomento e a ampliação de conhecimento que embasem a profissão em suas dimensões de assistência e melhora das condições de vida da população.

\section{CONCLUSÃO}

A realização do estudo possibilitou um conhecimento amplo, porém inicial, acerca do panorama de cursos de graduação em terapia ocupacional nos países da América Latina. Assinala-se a necessidade de ampliação e aprofundamento dos dados, bem como de sua análise, para maior exatidão do panorama em vigência, assim como possibilitar traçar estratégias coletivas para a área.

O baixo número de cursos de graduação em terapia ocupacional encontrado, sendo alguns de caráter técnico, pode ser um indicador da insuficiência de profissionais da área na região latino-americana. Entretanto, observase um significativo crescimento e aperfeiçoamento dos cursos nas duas últimas décadas, apontando para uma maior consolidação da profissão na região.

\section{REFERÊNCIAS}

1. Lillo SG, Blanche EI. Desarrollo de la terapia ocupacional en Latinoamérica. Rev Chilena Ter Ocup. 2010;10(1):123-35. doi: 10.5354/0717-5346.2010.10566.

2. Testa D. Aportes para el debate sobre los inicios de la profesionalización de la terapia ocupacional en Argentina. Rev Chilena Ter Ocup. 2012;12(1):67-78. doi: 10.5354/07175346.2012.22054.

3. Briglia J, Sartirana AG. Aspectos contextuales del surgimento de Terapia Ocupacional en la Argentina. Rev Chilena Ter Ocup. 2013;13(1):33-41. doi: 10.5354/07175346.2013.27450.

4. Moreno AF. Publicaciones seriadas de la terapia ocupacional en Latinoamerica. Cad Ter Ocup UFSCar. 2012;20(2):28792. doi: $10.4322 /$ cto. 2012.029 .

5. Lopes RE. Terapia ocupacional em São Paulo: um percurso singular e geral. Cad Ter Ocup UFSCar. 2004;12(2):75-88. Disponível em: http://www.cadernosdeterapiaocupacional. ufscar.br/index.php/cadernos/article/view/181.

6. Palm RCM, Oliver FC, Toldrá RC. VI Encontro LatinoAmericano de Escolas e Docentes de Terapia Ocupacional.
O campo da saúde com ênfase em aspectos biomédicos ainda constitui-se como um lugar prioritário para a atuação terapêutica-ocupacional. Faz-se necessário, contudo, questionar a emergência da questão social contemporânea ${ }^{25}$ e o real preparo dos profissionais para lidarem com intervenções direcionadas ao contexto latino-americano, que demanda profissionais envolvidos com a sua realidade social. Portanto, aponta-se a necessidade de aprofundamento de formação e estudos acerca do campo social como área de relevância para a formação profissional, seja qual for o setor de atuação do terapeuta ocupacional (saúde, educação, assistência social, cultura, justiça, trabalho etc).

Desenha-se como desafio para a terapia ocupacional latino-americana a implantação de novos cursos de graduação, o aumento do número de profissionais para a conquista de campos de atuação, o aumento das ofertas de pós-graduação stricto sensu específica em terapia ocupacional, o desenvolvimento de pesquisas e a construção de conhecimentos próprios que sustentem sua prática, contribuindo, conjuntamente, para a institucionalização da profissão na América Latina. A terapia ocupacional precisa caminhar na constituição de seus referenciais teóricos e metodológicos congruentes às demandas e realidades dos países da América Latina.

Rev Ter Ocup Univ São Paulo. 2011;22(3):i. doi: 10.11606/ issn.2238-6149.v22i3p298-307.

7. Palm RCM. Catálogo Latinoamericano de Asociaciones, Carreras y Postgrados de Terapia Ocupacional. Curitiba; 2012. Disponível em: http://www.crefito5.org.br/wpcontent/uploads/2012/04/Catalogo_Asociaciones_Carreras_ Postgrados-de-Terapia-Ocupacional_2011..pdf.

8. Bianchi PC. Terapia ocupacional e a questão social: retratos da formação graduada a partir de um recorte latino-americano [Dissertação]. São Carlos: Programa de Pós-graduação em Terapia Ocupacional, Universidade Federal de São Carlos; 2016. Disponível em: https:// repositorio.ufscar.br/bitstream/handle/ufscar/7531/ DissPCB.pdf? sequence $=1 \&$ isAllowed $=\mathrm{y}$.

9. Guajardo A. Chile. Terapia ocupacional. Apuntes para una historia inconclusa. In: Santos V, Gallassi AD, organizadores. Questões contemporâneas da terapia ocupacional na América do Sul. Curitiba: CRV; 2014. p.51-71.

10. Bianchi PC, Malfitano APS. A área social e a produção da revista chilena de terapia ocupacional: reflexões a partir 
da terapia ocupacional social. Rev Chilena Ter Ocup. 2016;16(2):127-41. doi: 10.5354/0717-6767.2016.44757.

11. Chiroleu A. Democratización universitaria y desigualdad social en América Latina. Política Universitária (Buenos Aires). 2014;1(1):26-31. Disponível em: http://iec.conadu. org.ar/files/publicaciones/1417195606_1-pu-web-pagsimple.pdf.

12. Figueiredo GV. Financiamento da educação: recursos necessários, fontes e o impacto das atuais propostas. Debate da Associação dos Docentes da Universidade Federal de Sâo Carlos - ADUFSCar Sindicato, em 27 jun 2016 [citado 05 ago. 2016]. Disponível em: https://www.youtube.com/ watch $\mathrm{v}_{\mathrm{v}}=\mathrm{C} 2 \mathrm{O} 31 \mathrm{sj} 9 \mathrm{oKI} \&$ spfreload $=10$.

13. Brasil. Ministério da Educação. Reuni - Reestruturação e expansão das universidades federais: diretrizes gerais. Brasília; 2007. Disponível em: http://portal.mec.gov.br/sesu/ arquivos/pdf/diretrizesreuni.pdf.

14. Pan LC. Políticas de ensino superior, graduação em terapia ocupacional e o ensino de terapia ocupacional social no Brasil [Dissertação]. São Carlos: Programa de Pós-graduação em Terapia Ocupacional, Universidade Federal de São Carlos; 2014. Disponível em: https://repositorio.ufscar.br/bitstream/ handle/ufscar/6889/5862.pdf?sequence=1\&isAllowed=y

15. Malfitano APS. Escassez de cursos de terapia ocupacional no Brasil. Audiência pública na Câmara dos Deputados em 16 jun 2016 [citado 05 ago. 2016]. Disponível em http:// www2.camara.leg.br/atividade-legislativa/webcamara/ videoArquivo codSessao $=57055 \#$ videoTitulo .

16. Durham ER, Sampaio H. O setor privado de ensino superior na América Latina. Cad Pesqui. 2000;110(s/n):7-37. Disponível em: http://www.scielo.br/pdf/cp/n110/n110a01.pdf.

17. Barba PDB, Silva RF, Joaquim RHVT, Brito CMD. Formação inovadora em Terapia Ocupacional. Interface (Botucatu). 2012;16(42):829-42. doi: 10.1590/S141432832012000300019 .

18. Hiratuka E, Duque AM, Andrade FL, Silva MNS, Menta SA, Soares TBA. Metodologias ativas como estratégias de ensino: experiência da terapia ocupacional na UFS. Cad Ter Ocup UFSCar. 2014;22(supl. esp.):487-92. Disponível em: http:// docplayer.com.br/12562779-Metodologias-ativas-comoestrategias-de-ensino-experiencia-da-terapia-ocupacionalna-ufs.html.

19. Conselho Nacional de Desenvolvimento Científico e Tecnológico (CNPq). Plataforma Lattes [citado 05 ago. 2016]. Disponível em: http://lattes.cnpq.br/.

20. Lopes RE, Malfitano APS, Oliver FC, Sfair SC, Medeiros T. Pesquisa em terapia ocupacional: apontamentos acerca dos caminhos acadêmicos no cenário nacional. Rev Ter Ocup Univ São Paulo. 2010;21(3):207-14. doi: 10.11606/ issn.2238-6149.v21i3p207-214.

21. Emmel MLG. Os caminhos da divulgação de conhecimento em terapia ocupacional no Brasil: o papel dos Cadernos de Terapia Ocupacional da UFSCar. Cad Ter Ocup UFSCar. 2015;23(3):449-50. doi: 10.4322\%2F0104-4931.ctoED2303.

22. Universidade Federal de Minas Gerais. Escola de Educação Física, Fisioterapia e Terapia Ocupacional (EEFFTO). Programa Pós-Graduação em Ciências da Reabilitação (PPGCR) [citado 23 mar. 2017]. Disponível em: http:// www.eeffto.ufmg.br/eeffto/pos_graduacao/ciencias_da reabilitacao_mestrado_doutorado.

23. Universidade de São Paulo (USP). Faculdade de Medicina, Departamento de Fisioterapia, Fonoaudiologia e Terapia Ocupacional (FOFITO). Programa de Pós-graduação em Ciências da Reabilitação [citado 23 mar. 2017]. Disponível em: http://www2.fm.usp.br/ppgreab/mostrahp. php? origem $=$ ppgreab $\&$ xcod $=$ HIST $\%$ D3RICO $\% 20 D O \% 20$ PROGRAMA\&dequem=HIST\%D3RICO.

24. Malfitano APS, Matsukura T, Martinez C, Emmel M, Lopes R. Programa de pós-graduação stricto sensu em terapia ocupacional: fortalecimento e expansão da produção de conhecimento na área. Rev Bras Atividade Física Saúde. 2013;18(1):105-11. doi: 10.12820/RBAFS.V.18N1P105-111.

25. Castel R. As metamorfoses da questão social: uma crônica do salário. Petrópolis: Editora Vozes; 1998.

Recebido em: 04.09.16

Aceito em: 20.04.17 